\title{
Chapter 4 \\ Metal Ions Introduced to Proteins \\ by Supramolecular Ligands
}

\author{
Olga Woźnicka, Joanna Rybarska, Anna Jagusiak, Leszek Konieczny, \\ Barbara Stopa, and Irena Roterman
}

\begin{abstract}
Congo red and other supramolecular structures may intercalate various foreign compounds, particularly planar ones. Such hybrid ligands, acting as a unit, may attach themselves to proteins and penetrate into their interior, together with any intercalated substances. If the intercalant is a metal complexone, a stable metalloprotein may be formed. This chapter discusses intercalation of metal complexones with metal ions bound by supramolecular Congo red as a means of introducing contrast to amyloid-like aggregates in order to trace the initial stages of amyloidogenesis. We investigate the applicability of Titan yellow carrying silver ions, and the alizarin complexone carrying tungsten and lead ions.
\end{abstract}

Keywords In vitro formed metalo-proteins • Supramolecular systems as metal ions carriages - Congo red and metal ions incorporation to proteins $\bullet$ Metal bearing supramolecular EM stains • Immune-complexes as Congo red target • Amyloid-like aggregates $\bullet$ EM contrast bearing Congo red $\bullet$ Metal ion complexation $\bullet$ Congo red and beta amyloid fibrils stained - Supramolecular systems and metal ion complexation

\footnotetext{
O. Woźnicka $(\bowtie)$

Department of Cell Biology and Imaging, Jagiellonian University,

Gronostajowa 9, 30-387 Krakow, Poland

e-mail: olga.woznicka@uj.edu.pl

J. Rybarska • A. Jagusiak • L. Konieczny • B. Stopa

Chair of Medical Biochemistry, Jagiellonian University - Medical College,

Kopernika 7, 31-024, Krakow, Poland

e-mail: mbstylin@cyf-kr.edu.pl; anna.jagusiak@uj.edu.pl; mbkoniec@cyf-kr.edu.pl;

barbara.stopa@uj.edu.pl

I. Roterman

Department of Bioinformatics and Telemedicine, Jagiellonian University - Medical College,

Łazarza 16, 31-530, Krakow, Poland

e-mail: myroterm@cyf-kr.edu.pl
} 


\subsection{Metal Ions in Natural Biological Systems}

Many proteins, including enzymes, rely on metal ions for their biological activity. The ions themselves are usually transition metals, such as iron, cobalt, nickel or copper. They enable catalysis due to their electron structure and the ability to form coordinate bonds. Another commonly encountered metal is zinc - it can be found e.g. in proteolytic enzymes known as metalloproteinases whose peculiar complexation capabilities have attracted much scientific attention.

Nearly one-third of all known enzymes include some type of metal ion. Metals are primarily associated with catalysis, but they also play an important part in formation of specific complexes, such as oxygen binding - e.g. in hemoglobin, which contains iron. Metals are also encountered in transcription factors, and along many other biochemical pathways, such as respiration. Certain protein complexes are dedicated to sequestration and/or accumulation of metals (e.g. siderophilin and ferritin) as well as detoxification (e.g. metallothionein).

Transition metals are sometimes referred to as "d"-electron metals due to the involvement of their " $\mathrm{d}$ " orbitals in atomic interactions. They form a variety of compounds with interesting spectroscopic and magnetic properties whose practical applications are the subject of ongoing research [1-17]. In the cell, metals are usually found in their complexed form, either as standalone ions or inside specific planar carrier compounds encapsulated by proteins - e.g. the porphyrin ring in hemoglobin. Proteins provide the capability to bind metals, isolate them from water and may introduce favorable steric conditions [18]. A classic example is hemoglobin, which enables oxygen binding without oxidation of the bivalent iron. Combining metal ions with proteins enhances their catalytic potential. The search for artificial structures with desirable catalytic properties is an important topic in medical science. The bond between the metal and the protein should not be random in character, since such uncontrolled complexation is usually encountered on the protein surface, where the metal remains in contact with water. In order to reduce polarity, the ion should optimally be placed right in the pocket of the active group. In practice, however, this is a very challenging task. An interesting solution is proposed in [19], where a metal ion was attached to streptavidin by connecting the Ru complex with biotin via a carboxyl group. This resulted in catalytic activity even though the complexed ion was located nearly on the surface (the biotin binding cavity has a depth of approximately $15 \AA$, which coincides with the length of the complex) [20].

\subsection{Insertion of Metal Ions into Proteins by Supramolecular Ligands}

An entirely different approach to binding metals with proteins relies on supramolecular ligands. Such ligands can form stable complexes with proteins which have become susceptible to penetration as a result of function-related structural changes, 
or which possess such properties natively [21-23]. Certain proteins can be induced to undergo complexation with supramolecular ligands e.g. by heating, which causes partial unfolding of the polypeptide chain. However, only ribbonlike supramolecular structures are capable of penetrating into proteins and forming stable complexes. Examples include CR, EB and others [24, 25] - dyes with known affinity for amyloids and immune complexes [26]. Supramolecular systems (e.g. CR) may, in turn, intercalate a variety of planar compounds with a polyaromatic ring structure and/or positive charge. Hence if the intercalant itself contains a metal ion, the ion can be attached to the protein. As a result supramolecular ligands can bind metal ions to proteins by intercalating their complexons.

The supramolecular ligand usually binds outside of the protein's active site. The complex is formed in the distal part of the molecule, however the ligand penetrates into the protein interior, where polarity is lower and no water is present - similarly to the active site $[22,27,28]$. Exposure of the intercalated metal ion depends on the structure of the resulting complex. This means that attaching metals to proteins seems at the moment more convenient than through the use of customized substrates or enzymatic inhibitors. The ion is not delivered to the active site, but the resulting conformation shares some similarities with the structure of the active site, and may possess useful biological properties.

The goal of our team was to confirm the proposed means of attaching metals to proteins, and also to devise a way to equip $\mathrm{CR}$ - a known amyloid stain - with contrast for the purposes of EM imaging. The complexation of $\mathrm{CR}$ with amyloids is the subject of numerous studies [29-31]. The problem is difficult and its molecular underpinnings remain speculative, since CR itself is not visualized under electron microscopy, while amyloids - despite their ordered structure - do not attain the necessary level of crystallization order. One putative solution would be to add contrast to $\mathrm{CR}$ itself to visualize its distribution with respect to amyloid deposits. Assuming that $\mathrm{CR}$ binds to amyloids as a supramolecular system, the contrast could be introduced as a metal-containing intercalant. The proposed compound - TY comprises symmetric polar groups and aromatic rings [32, 33] (Fig. 4.1). Its halves are linked by a tri-azo bond capable of complexing metal ions, particularly silver and mercury. The silver-containing complex is more convenient due to its stability and formation in both neutral and slightly alkaline environments. TY/ $\mathrm{Ag}^{+}$complexation is also easy to detect since its spectrum differs markedly from the spectrum of free TY (Fig. 4.1).

The complex withstands electrophoretic dissociation in alkaline $\mathrm{pH}$ in a tris buffer (Fig. 4.2). It dissociates in the presence of anions, yielding insoluble silver compounds (solubility coefficient $<10^{-13}$ ), which indicates that in biological systems only thiol groups are effectively able to react with silver bound in the complex. A certain disadvantage of this complex is its notable viscosity. This is due to the specific mode of interaction between the silver ion and the tri-azene bond - it may involve both coordination valence electrons of silver ion, or only one, if the other has easy access to the tri-azene group of another dye molecule. This phenomenon promotes association of complexes, particularly at high dye concentrations. Fortunately, the viscosity of TY/ $\mathrm{Ag}^{+}$complexes is greatly decreased in the presence 


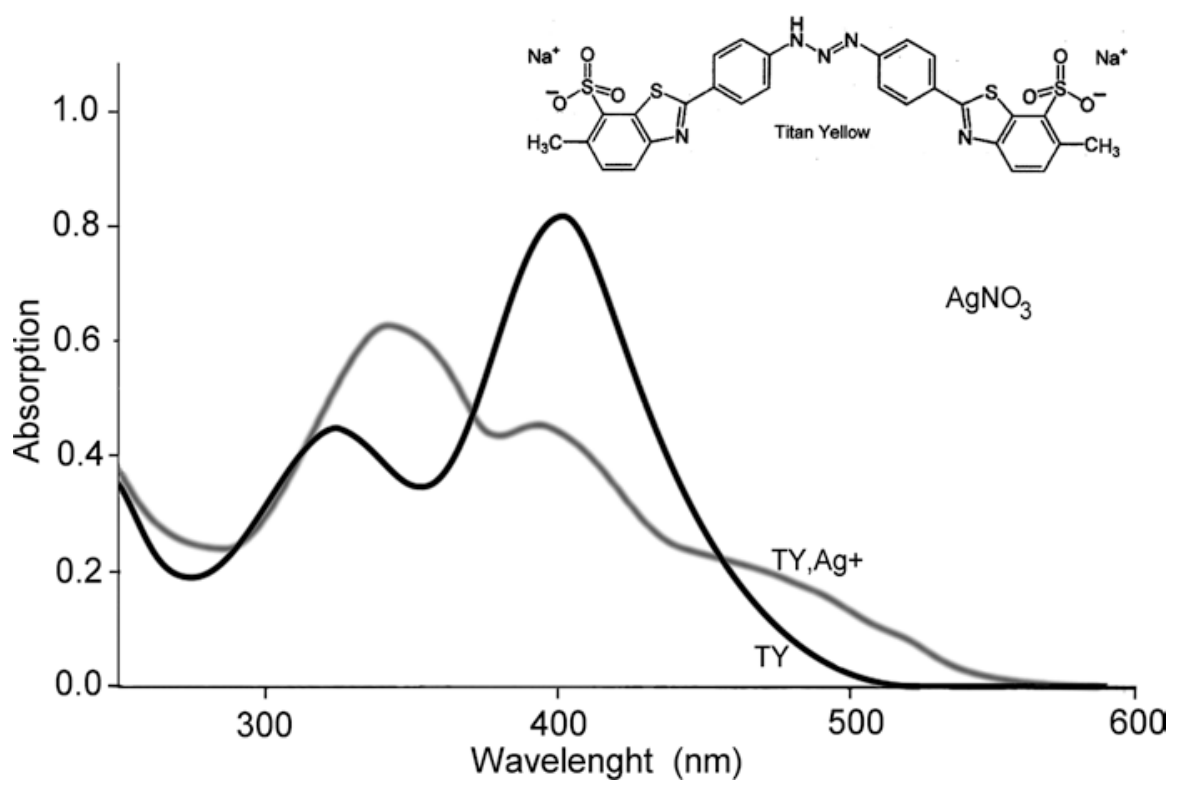

Fig. 4.1 Spectral changes effect of silver ion complexation with TY Inset: the formula of TY

Fig. 4.2 Agarose electrophoresis of dyes used for staining amyloids and amyloid-like aggregates. Accelerated migration of CR mixed with TY is the evidence of mutual complexation. $1-\mathrm{TY} / \mathrm{Ag}^{+}, 2-\mathrm{TY}$, $3-\mathrm{CR} / \mathrm{TY}, 4-\mathrm{CR}$ 
of CR, whose molecules separate the intercalants, preventing mutual interactions. Intercalation of TY enhances the contrast characteristics of $\mathrm{CR}$, rendering it useful for EM imaging of amyloids.

Initial research involving contrast-enhanced $\mathrm{CR}$ has been carried out with amyloid-like aggregations formed by shaking of IgG light chains at increased temperatures $\left(40-45{ }^{\circ} \mathrm{C}\right.$ ), following which the precipitate was treated with the CR$\mathrm{TY}-\mathrm{Ag}^{+}$complex. The progressive appearance of structural order can be observed by imaging CR, which is a selective amyloid stain. In the presented case, aggregations were formed by shaking an amyloidogenic protein (IgG light chain) in conditions verging on denaturation: increased temperature and $\mathrm{pH}$ inconsistent with the protein's isoelectric point. These conditions promote the formation of amorphous aggregations in which seeds of order randomly emerge - typically as short, spiraling threads capable of binding the supramolecular dye. Such ordering is easier to observe and analyze along the edges of solids and/or in fine flecks of aggregates. The contrast medium carried by CR greatly simplifies molecular analysis.

Figure 4.3 presents the contrast-enhanced aggregate as seen under an electron microscope. Accurate interpretation of results requires however carefully prepared samples and an optimized method - still, even at this early stage it seems evident that the image carries useful information. Functional optimization should involve formation and stability of the protein-metal complex, but also even distribution of $\mathrm{CR}$ and the intercalated contrast medium. A classic amyloid aggregation formed by commercially available peptides (amyloid beta) is shown in Fig. 4.4 - it appears as twisted strands whose thickness depends on the synthesis procedure.

The proposed approach may also be used to stain antibodies engaged in immune complexes, since immune complexation renders antibodies susceptible to penetration by CR [34, 35]. Figure 4.5 presents sheep red blood cell membranes (ghosts) broken to pieces by homogenization and then agglutinated by specific antibodies obtained from the sensitized rabbit (anti-SRBC serum).

Another, more universal complexon analyzed in the search for convenient methods of attaching metal ions into proteins is the alizarincomplexone (Fig. 4.6). It fulfills all the necessary conditions - it can bind a variety of metals and is itself intercalated by $\mathrm{CR}$.

Unfortunately, it requires acidic conditions, since metal complexation is mediated by two acetic acid residues (which undergo ionization in a neutral or basic environment, declining their ability to bind the metal). The problem is additionally exacerbated by the fact that in $\mathrm{pH}<5 \mathrm{CR}$ transitions into a chinoid compound, becoming insoluble. Taken together, these two phenomena establish a rather narrow $\mathrm{pH}$ range where effective complexation may take place. The alizarincomplexone forms complexes with cobalt, lead, nickel and with the salts of certain other metals such as molybdenum and nickel. Complexation is evidenced by spectral changes (Figs. 4.7 and 4.8). Complexes persist under electrophoresis. 


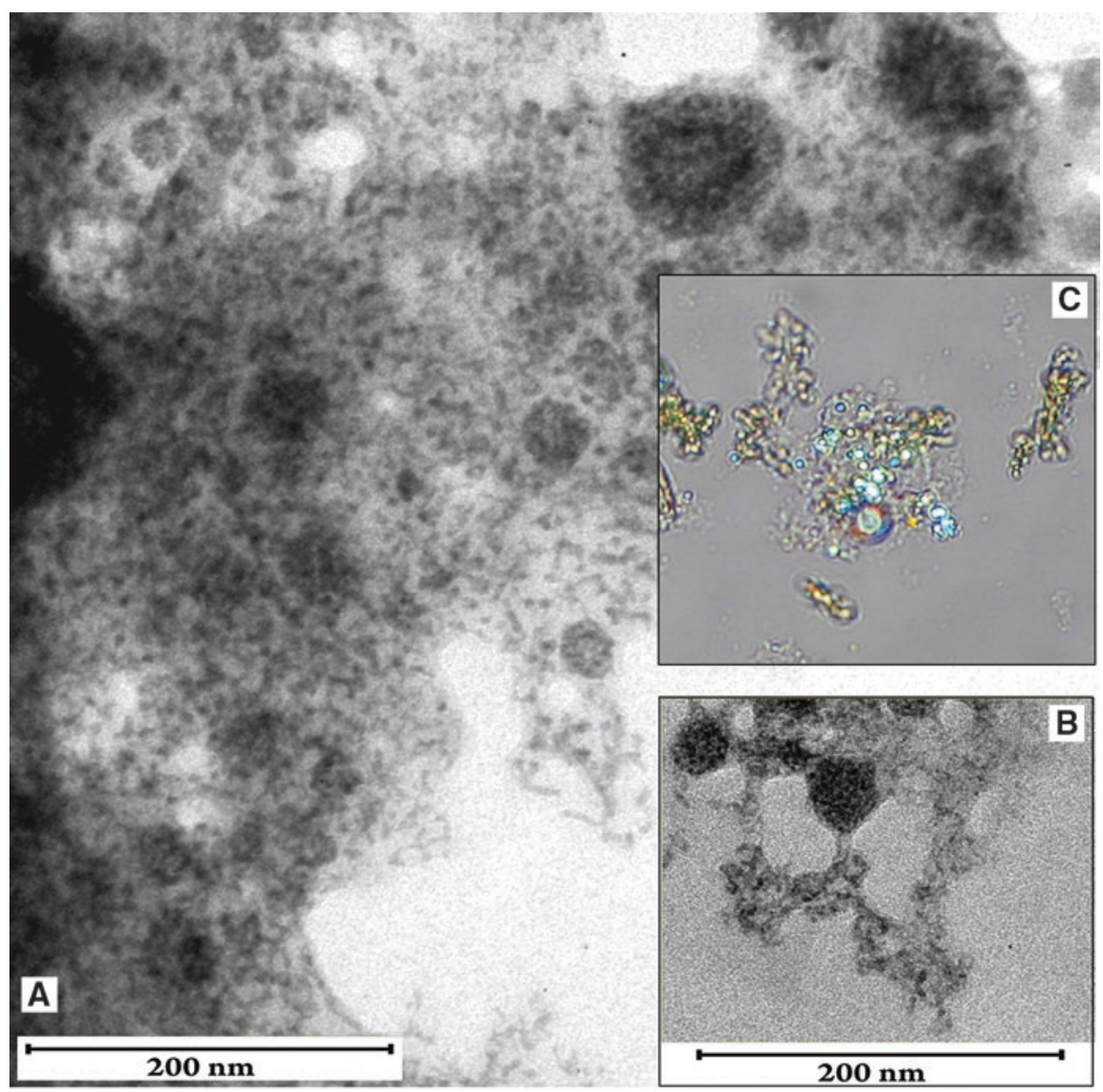

Fig. 4.3 Amyloid-like particles. (A and B) The edge fragments contrasted with complex CR/TY/ $\mathrm{Ag}^{+}$used as the stain. (C) Aggregates seen in the polarised light

Tungsten and lead complexes may serve as a contrast medium when intercalated by $\mathrm{CR}$ in $0.05 \mathrm{M}$ acetate buffer $(\mathrm{pH}=5.9)$.

The resulting EM images reveal various modes of aggregation, depending on the target polypeptide chain and environmental conditions. In most cases the aggregate is amorphous but contains islands of ordered structures, seen as granular chains of varying length, with longer chains often spirally twisted. When viewed in polarized light, these ordered fragments exhibit birefringence and glow, indicating that they constitute of ordered amyloid precursors (Figs. 4.9, 4.10, 4.11 and 4.12). Nevertheless, a detailed description of their structure requires further research. 
Fig. 4.4 Amyloid Beta 1-40 (Sigma) derived fibrils stained by $(\mathrm{CR} / \mathrm{TY} /$ $\mathrm{Ag}^{+}$) complex seen in EM picture. Supplement picture below - the enlargement of fibrils

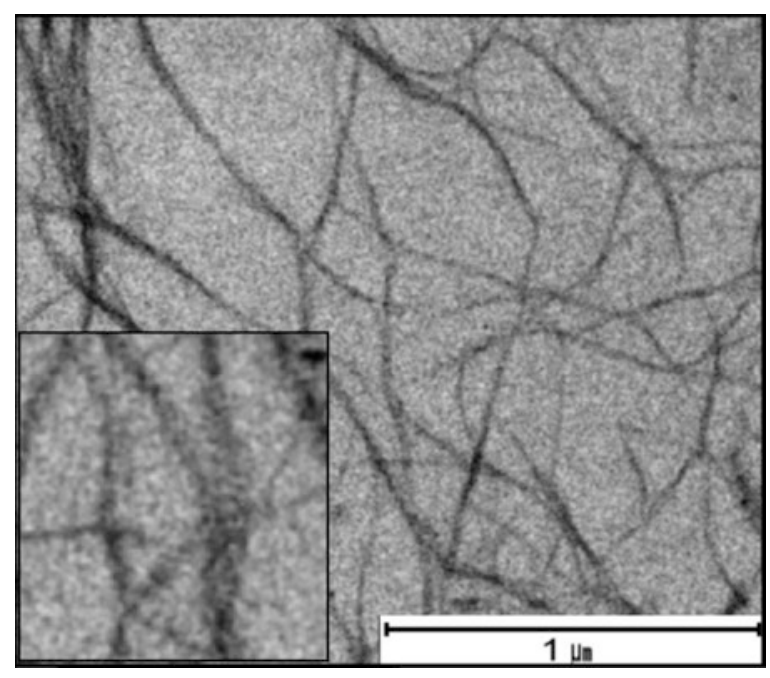

\subsection{Conclusions}

Transition metal ions exhibit complexation capabilities which may be of significant practical importance. Biological applications focus on introduction of such ions into proteins, where they exhibit greater reactivity than in their unbound form. The role of the protein in this process is not entirely clear, although changes in environmental factors (particularly reduction in polarity) appear to play an important role. Under these assumptions, it is not necessary for the metal ion to be placed in the active site, since this is usually not the only area characterized by low polarity. Internalization of metal ions is facilitated by supramolecular ligands, capable of penetrating into proteins in unstable areas basically other than the active site. Such ligands can serve as carriers for a variety of organic molecules, including those which contain metal ions. The metal-containing compound is attached to the ligand via intercalation, which naturally leads us to search for suitable metal complexones, able to form strong bonds with CR. Examples include TY and the alizarin complexone. Confirmation of the metal-protein bond is provided by microscopic imaging of amyloid-like aggregations formed by the IgG light chain stained by CR in complex with TY/silver ions.

EM images reveal amorphous aggregations which contain ordered structures capable of binding the contrast medium. Such structures typically adopt the form of 


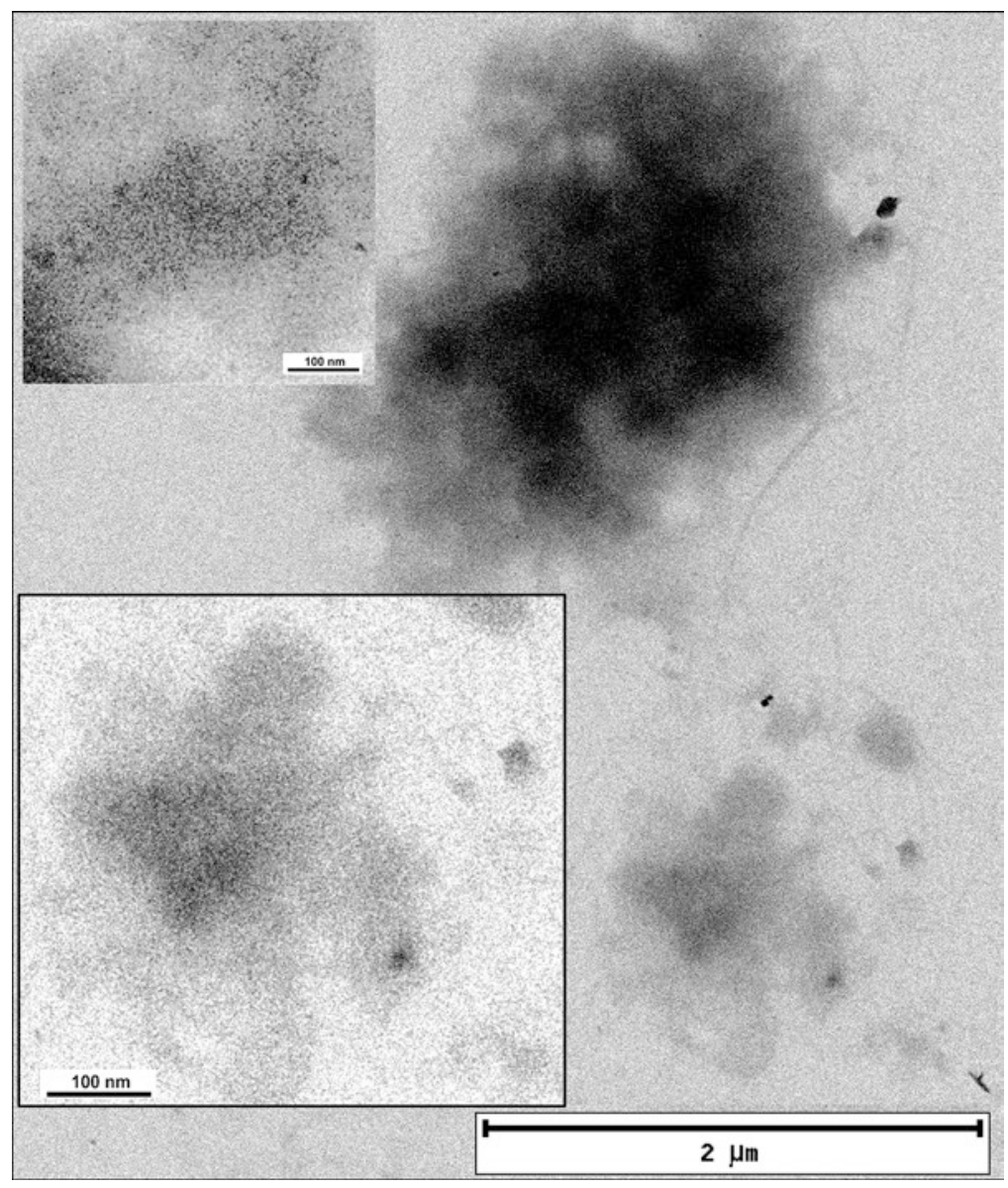

Fig. 4.5 Agglutinates of fragmented by homogenisation erythrocyte ghosts (SRBC) stained by $\mathrm{CR} / \mathrm{TY} / \mathrm{Ag}^{+}$. Insets - enlarged fragments

twisted strands and function as seeds for amyloid transformation. In addition to bulk solids (which are difficult to study with EM), some aggregates also adopt the form of thin membranes, exposing the contrast medium and confirming that supramolecular ligands may successfully attach metal ions to proteins. Thus, the location of CR concentrations in microscopic images reveals the shape of the analyzed object. While amyloids are known to adopt various structures, depending on the conformation of the unit protein and on environmental conditions, all such structures retain the ability to bind CR (Figs. 4.7, 4.8, 4.9 and 4.10) show fragments of amyloid-like aggregates with portions containing ordered structures exposed by contrast. As can be seen, the bulk of the aggregate remains amorphous and incapable of 


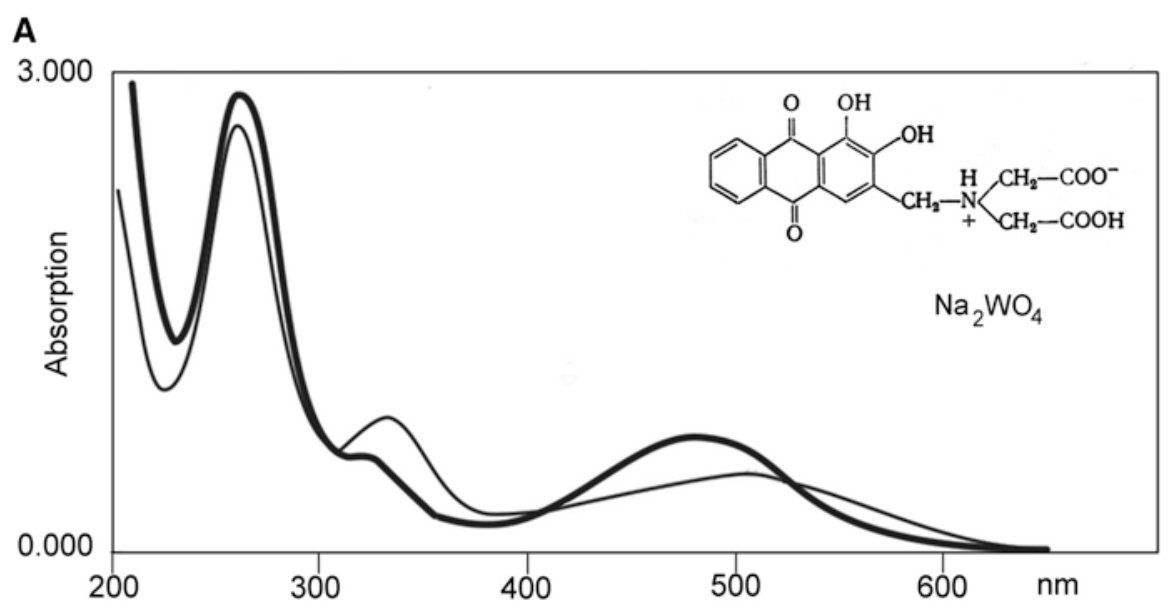

B

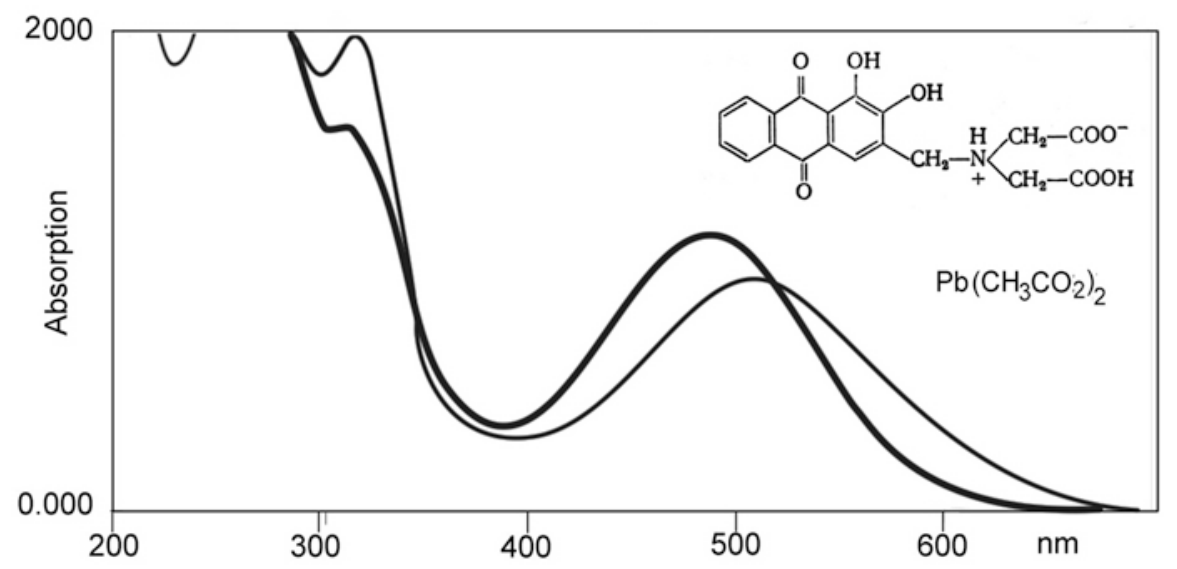

Fig. 4.6 Spectral changes associating the complexation of alisarine complexon (AC) with tungstate ion $-\mathbf{A}$ and lead ion $-\mathbf{B}$

binding the dye. Due to its thickness, it produces some darkening in the resulting images, but even so the contrast is clearly discernible. The ordered forms constitute seeds for amyloid transformation, which explains their ability to bind CR.

Aggregation is mediated by light chain $\mathrm{V}$ domains, which are less stable than the corresponding $\mathrm{C}$ domains. Prior to aggregation, $\mathrm{V}$ domains undergo partial unfolding initiated by displacement of $\mathrm{N}$-terminal chain. This uncovers the interior of domains [21] making available a pocket in which the CR aggregate may anchor itself. It appears that unfolding affects the so-called upper core [36, 37] and occurs symmetrically in the dimeric structure of the protein. Partly unfolded V domains may subsequently form beta-beta bonds with adjacent molecules, creating a fibril. 

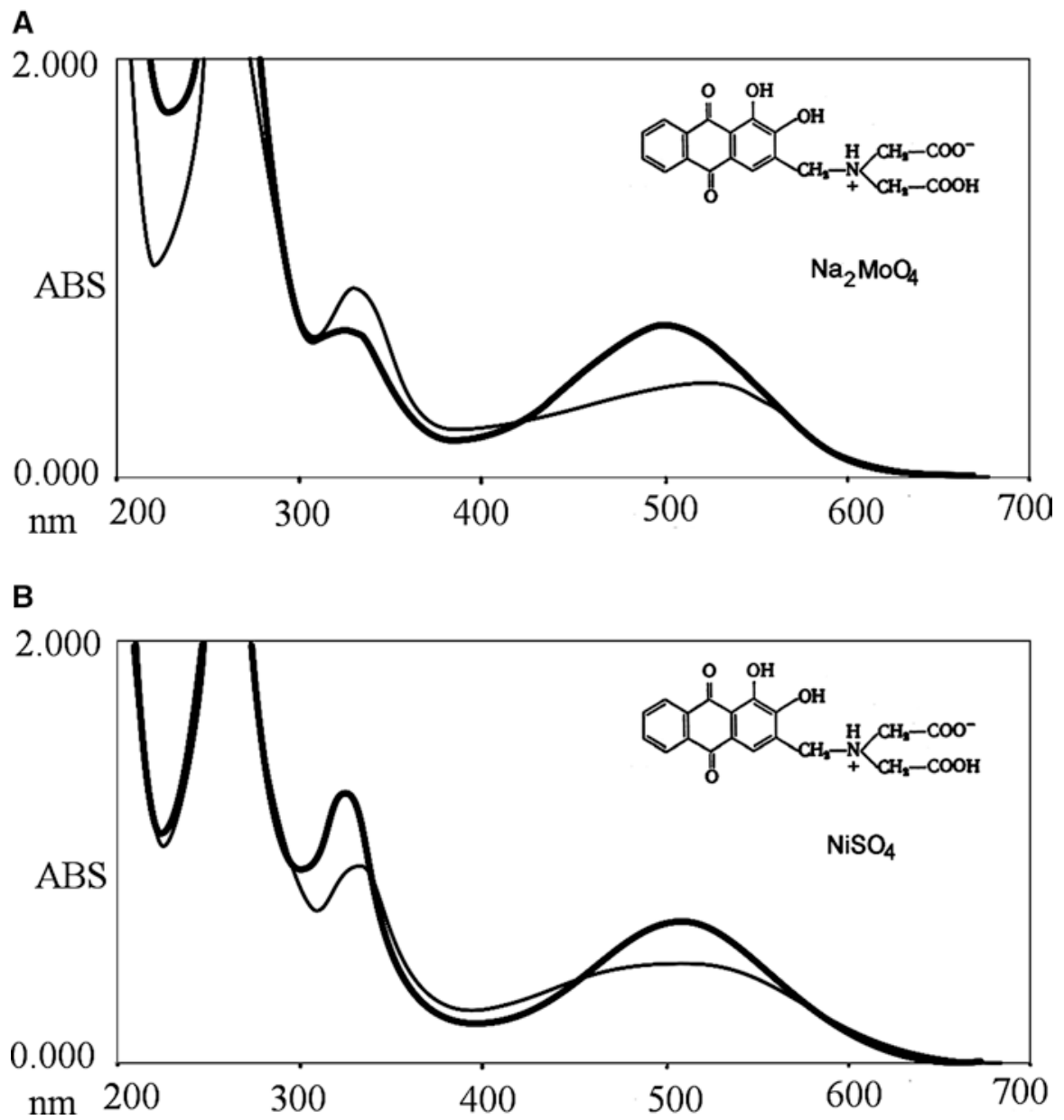

Fig. 4.7 Spectral changes presented as an evidence of alizarin complexon binding different metals - Mo (A) as molibdate $\mathrm{MoO}_{4}{ }^{2-}$ and $\mathrm{Ni}^{2+}(\mathbf{B})$

The role of the lower core (responsible for stabilization of the inter-chain interface) in this process is not clear, as is its involvement in structural rearrangements in the $\mathrm{V}$ domain. It seems that fibrils can form in either case; however, strand-like products are expected to possess different properties. Molecular interpretation of CR binding to amyloid fibrils formed by commercially available Amyloid Beta peptide 40 requires further study. In contrast to native proteins which undergo structural rearrangements facilitating penetration of a supramolecular ligand, the packing of Beta peptide 40 derived amyloid fibrils is tight and uniform. The dye may potentially penetrate in areas where the fibril is sheared or otherwise structurally disrupted. 


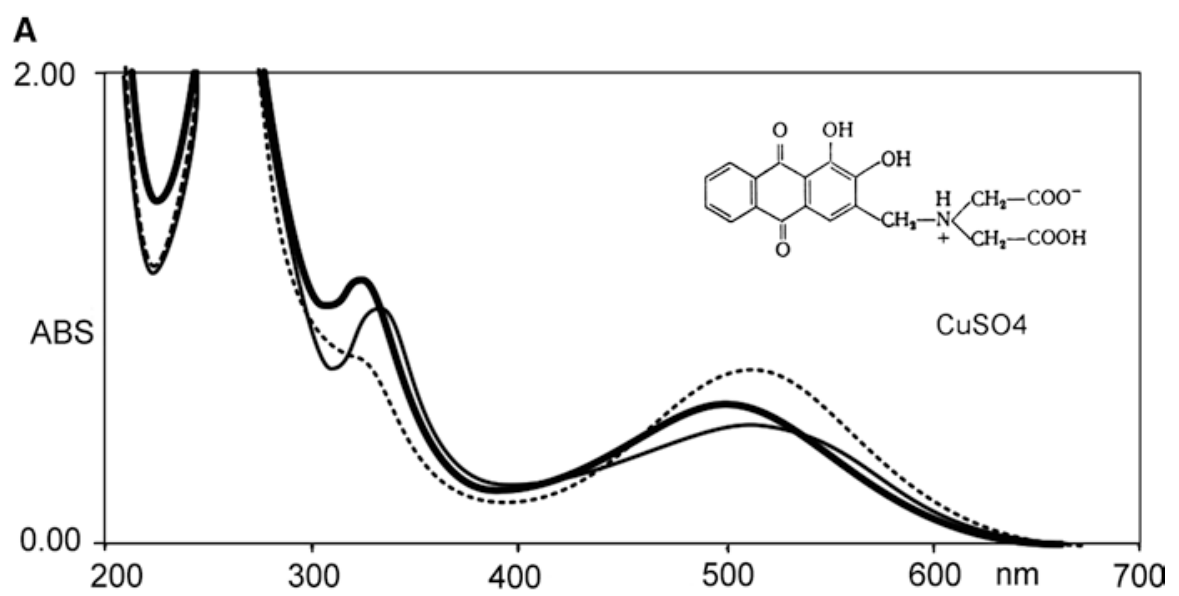

B

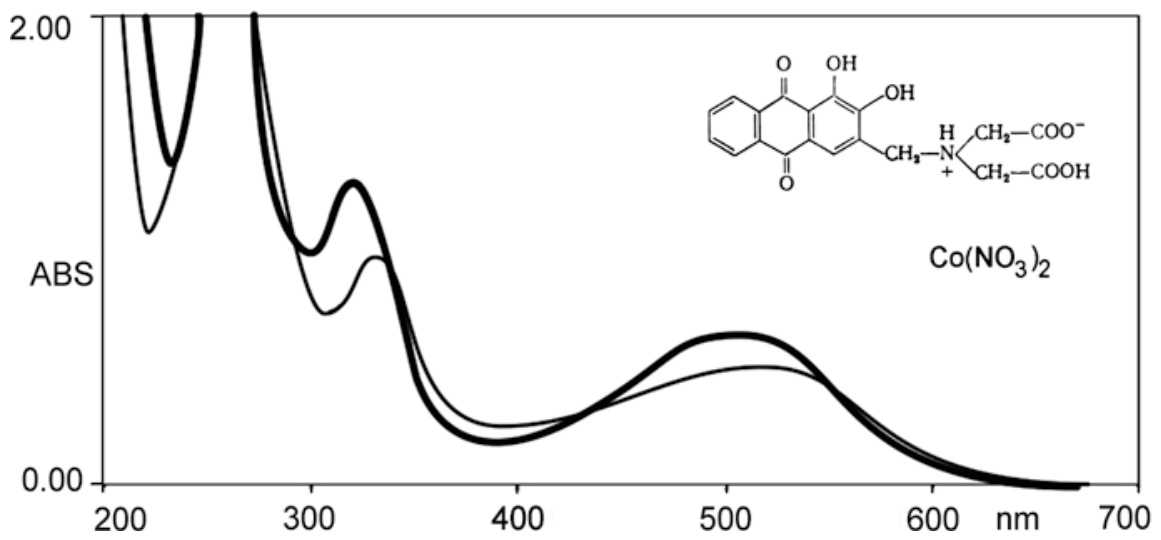

Fig. 4.8 Spectral changes presented as an evidence of alizarincomplexon binding ions of different metals $-\mathrm{Cu}(\mathbf{A})$ and $\mathrm{Co}(\mathbf{B})$. The spectrum change marked by dotted line indicates that $\mathrm{Cu}^{2+} \mathrm{com}-$ plexation reveals the formation of modified complex at the excess of $\mathrm{Cu}^{2+}$ ions added. Molar ratio of $\mathrm{AC}: \mathrm{Cu}^{2+} 1: 2$

Complexation is further hampered by the fact that fibrils often consist of multiple intertwined strands - in such cases the ligand may engage individual strands without disrupting the fibril as a whole. This theory is supported by EM analysis, showing that $\mathrm{CR}$ does not uniformly cover the entire fibril, despite an abundance of the dye (Fig. 4.4), and that the fibril is unwound in many areas. It should also be noted that ribbon-like CR may attach itself to amyloids by means of adhesion.

Immune complexes are another type of object which can be visualized in the same manner - this is due to the fact that, by binding their natural ligand, antibodies 
Fig. 4.9 Islands of ordered structures formed within the amyloid-like particle stained selectively with $\mathrm{CR} / \mathrm{AC} / \mathrm{WoO}_{4}{ }^{2-}$ complex

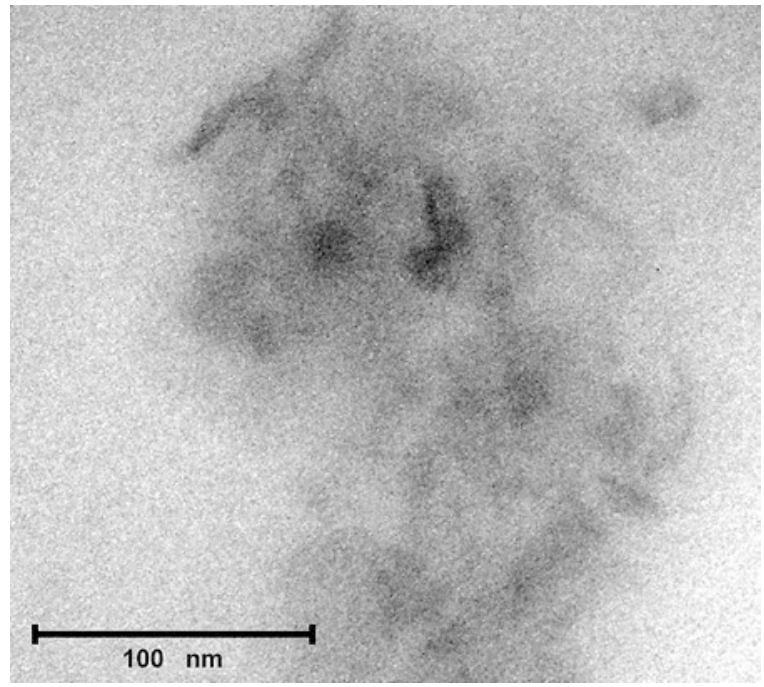

Fig. 4.10 Amyloid-like particles with ordered structures inside stained with $\mathrm{CR} / \mathrm{AC} / \mathrm{Pb}^{+2}$

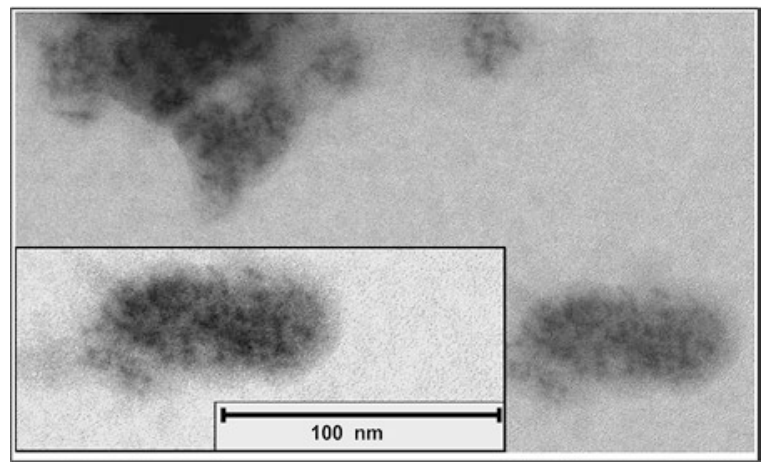

incur local structural instabilities which render them susceptible to penetration by supramolecular dyes, such as CR.

The proposed ligands and the procedure outlined in this work are both preliminary in scope. The technique can be further optimized by carefully selecting reagents and modifying the manner in which metal ions are transported by supramolecular ligands. 


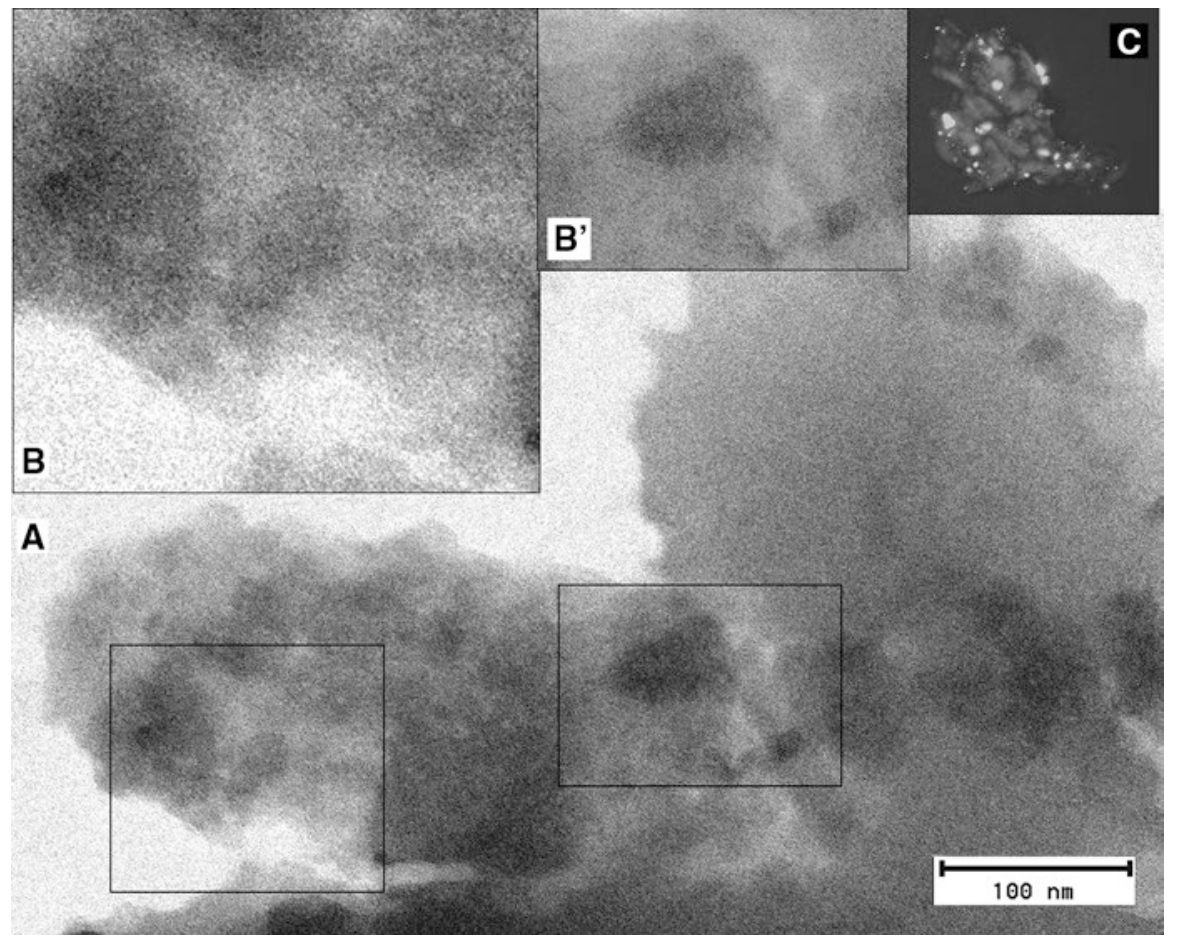

Fig. 4.11 Islands of ordered structure within amyloid-like particle stained selectively with (A) $\mathrm{CR} / \mathrm{AC} \mathrm{Pb}^{2+}$ complex used as the contrast for EM studies. (B and $\mathbf{B}^{\prime}$ ) - enlarged fragments, $(\mathbf{C})$ islands of ordered structures seen in amyloid-like particle in polarised light

Fig. 4.12 The ordered structures bearing fragment outgrowing unstructured amyloid particle stained by $\mathrm{CR} / \mathrm{AC} / \mathrm{Pb}^{+2}$. Local burst of ordered structures

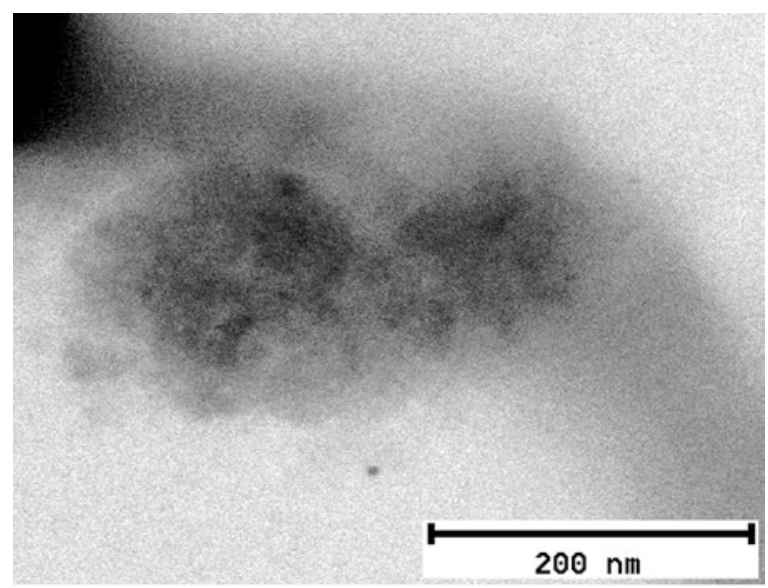


Acknowledgements We acknowledge the financial support from the National Science Centre, Poland (grant no. 2016/21/D/NZ1/02763) and from the project Interdisciplinary PhD Studies "Molecular sciences for medicine" (co-financed by the European Social Fund within the Human Capital Operational Programme) and Ministry of Science and Higher Education (grant no. K/ DSC/001370).

\section{References}

1. Sorenson JR, Wangila GW (2007) Co-treatment with copper compounds dramatically decreases toxicities observed with cisplatin cancer therapy and the anticancer efficacy of some copper chelates supports the conclusion that copper chelate therapy may be markedly more effective and less toxic than cisplatin therapy. Curr Med Chem 14(14):1499-1503

2. Begley TP (2017) Origin of a key player in methane biosynthesis. Nature 543(7643):49-50

3. Biersack B, Ahmad A, Sarkar FH, Schobert R (2012) Coinage metal complexes against breast cancer. Curr Med Chem 19(23):3949-3956

4. Cooper GJ (2012) Selective divalent copper chelation for the treatment of diabetes mellitus. Curr Med Chem 19(17):2828-2860

5. Zhang J, Zhang F, Li H, Liu C, Xia J, Ma L, Chu W, Zhang Z, Chen C, Li S, Wang S (2012) Recent progress and future potential for metal complexes as anticancer drugs targeting G-quadruplex DNA. Curr Med Chem 19(18):2957-2975

6. Wang T, Guo Z (2006) Copper in medicine: homeostasis, chelation therapy and antitumor drug design. Curr Med Chem 13(5):525-537

7. Ashfaq M, Najam T, Shah SS, Ahmad MM, Shaheen S, Tabassum R, Rivera G (2014) DNA binding mode of transition metal complexes, a relationship to tumor cell toxicity. Curr Med Chem 21(26):3081-3094

8. El Kazzouli S, El Brahmi N, Mignani S, Bousmina M, Zablocka M, Majoral JP (2012) From metallodrugs to metallodendrimers for nanotherapy in oncology: a concise overview. Curr Med Chem 19(29):4995-5010

9. Fanni D, Fanos V, Gerosa C, Piras M, Dessi A, Atzei A, Van EP, Gibo Y, Faa G (2014) Effects of iron and copper overload on the human liver: an ultrastructural study. Curr Med Chem 21(33):3768-3774

10. Gumienna-Kontecka E, Pyrkosz-Bulska M, Szebesczyk A, Ostrowska M (2014) Iron chelating strategies in systemic metal overload, neurodegeneration and cancer. Curr Med Chem 21(33):3741-3767

11. Inoue K, O'Bryant Z, Xiong ZG (2015) Zinc-permeable ion channels: effects on intracellular zinc dynamics and potential physiological/pathophysiological significance. Curr Med Chem 22(10): $1248-1257$

12. Kaluderović GN, Paschke R (2011) Anticancer metallotherapeutics in preclinical development. Curr Med Chem 18(31):4738-4752

13. Kellett A, Prisecaru A, Slator C, Molphy Z, McCann M (2013) Metal-based antimicrobial protease inhibitors. Curr Med Chem 20(25):3134-3151

14. Ruiz-Azuara L, Bravo-Gómez ME (2010) Copper compounds in cancer chemotherapy. Curr Med Chem 17(31):3606-3615

15. Tardito S, Marchiò L (2009) Copper compounds in anticancer strategies. Curr Med Chem 16(11):1325-1348 
16. Terreno E, Dastrù W, Delli Castelli D, Gianolio E, Geninatti Crich S, Longo D, Aime S (2010) Advances in metal-based probes for MR molecular imaging applications. Curr Med Chem 17(31):3684-3700

17. Ward RJ, Dexter DT, Crichton RR (2012) Chelating agents for neurodegenerative diseases. Curr Med Chem 19(17):2760-2772

18. Collman JP, Brauman JI, Rose E, Suslick KS (1978) Cooperativity in O2 binding to iron porphyrins. Proc Natl Acad Sci U S A 75(3):1052-1055

19. Jeschek M, Reuter R, Heinisch T, Trindler C, Klehr J, Panke S, Ward TR (2016) Directed evolution of artificial metalloenzymes for in vivo metathesis. Nature 537(7622):661-665

20. Green NM, Konieczny L, Toms EJ, Valentine RC (1971) The use of bifunctional biotinyl compounds to determine the arrangement of subunits in avidin. Biochem J 125(3):781-791

21. Piekarska B, Konieczny L, Rybarska J, Stopa B, Zemanek G, Szneler E, Król M, Nowak M, Roterman I (2001) Heat-induced formation of a specific binding site for self-assembled Congo Red in the V domain of immunoglobulin L chain lambda. Biopolymers 59(6):446-456

22. Roterman I, No KT, Piekarska B, Kaszuba J, Pawlicki R, Rybarska J, Konieczny L (1993) Bis azo dyes--studies on the mechanism of complex formation with IgG modulated by heating or antigen binding. J Physiol Pharmacol 44(3):213-232

23. Stopa B, Piekarska B, Konieczny L, Rybarska J, Spólnik P, Zemanek G, Roterman I, Król M (2003) The structure and protein binding of amyloid-specific dye reagents. Acta Biochim Pol 50(4):1213-1227

24. Król M, Roterman I, Piekarska B, Konieczny L, Rybarska J, Stopa B, Spólnik P, Szneler E (2005) An approach to understand the complexation of supramolecular dye Congo red with immunoglobulin L chain lambda. Biopolymers 77(3):155-162

25. Skowronek M, Stopa B, Konieczny L, Rybarska J, Piekarska B, Szneler E, Bakalarski G, Roterman I (1998) Self-assembly of Congo red - a theoretical and experimental approach to identify its supramolecular orhanization in water and salt solutions. Biopolymers 46:267-281

26. Rybarska J, Konieczny L, Roterman I, Piekarska B (1991) The effect of azo dyes on the formation of immune complexes. Arch Immunol Ther Exp (Warsz) 39(3):317-327

27. Piekarska B, Konieczny L, Rybarska J (1988) The effect of azo dyes on heat aggregation of IgG. Acta Biochim Pol 35(4):297-305

28. Konieczny L, Piekarska B, Rybarska J, Stopa B, Krzykwa B, Nowowrolski J, Pawlicki R, Roterman I (1994) Bis-azo dye liquid crystalline micelles as possibile drug carriers in immunotargeting technique. J Physiol Pharmacol 45:441-454

29. Frid P, Anisimov SV, Popovic N (2007) Congo red and protein aggregation in neurodegenerative disease. Brain Res Rev 53:135-160

30. Lendel C, Bolognesi B, Wahlstrőm A, Dobson CM, Gräslund A (2010) Detergent-like interaction of Congo red with the amyloid $\beta$-peptide. Biochemistry 49:1358-1360

31. Khurana R, Gillespie JR, Talapatra A, Minert LJ, Ionescu-Zanetti C, Millett I, Fink AL (2001) Partially folded intermediates as critical precursors of light chain amyloid fibrils and amorphous aggregates. Biochemistry 40:3525-3535

32. Lazny R, Poplawski J, Köbberling J, Anders D, Brësse S (1999) Triazenes: a useful protecting strategy for sensitive secondary amines. Synlett 8:1301-1306

33. King HGC, Pruden G (1967) The component of commercial titan yellow most reactive towards magnesium: Its isolation and use in determining magnesium in silicate minerals. Analyst 92:83-90

34. Rybarska J, Konieczny L, Roterman I, Piekarska B (1991) The effect of Azo dyes on the formation of immune complexes. Archiv Immunol Ther Exp 39:317-327 
35. Jagusiak A, Konieczny L, Krol M, Marszalek P, Piekarska B, Piwowar P, Roterman I, Rybarska J, Stopa B, Zemanek G (2015) Intramolecular immunological signal hypothesis revived structural background of signalling revealed by using Congo red as a specific tool. Mini Rev Med Chem 14(13):1104-1113

36. Ewert S, Honegger A, Plückthun A (2004) Stability improvement of antibodies for extracellular and intracellular applications: CDR grafting to stable frameworks and structure-based framework engineering. Methods 34(2):184-199

37. Röthlisberger D, Honegger A, Plückthun A (2005) Domain interactions in the Fab fragment: a comparative evaluation of the single-chain $\mathrm{Fv}$ and $\mathrm{Fab}$ format engineered with variable domains of different stability. J Mol Biol 347(4):773-789

Open Access This chapter is distributed under the terms of the Creative Commons Attribution 4.0 International License (http://creativecommons.org/licenses/by/4.0/), which permits use, duplication, adaptation, distribution and reproduction in any medium or format, as long as you give appropriate credit to the original author(s) and the source, provide a link to the Creative Commons license and indicate if changes were made.

The images or other third party material in this chapter are included in the work's Creative Commons license, unless indicated otherwise in the credit line; if such material is not included in the work's Creative Commons license and the respective action is not permitted by statutory regulation, users will need to obtain permission from the license holder to duplicate, adapt or reproduce the material. 